\title{
Expression and Preliminary Functional Profiling of the let-7 Family during Porcine Ovary Follicle Atresia
}

\author{
Rui Cao, Wang Jun Wu, Xiao Long Zhou, Peng Xiao, Yi Wang, and Hong Lin Liu*
}

\begin{abstract}
Most follicles in the mammalian ovary undergo atresia. Granulosa cell apoptosis is a hallmark of follicle atresia. Our previous study using a microRNA (miRNA) microarray showed that the let-7 microRNA family was differentially expressed during follicular atresia. However, whether the let7 miRNA family members are related to porcine (Sus scrofa) ovary follicular apoptosis is unclear. In the current study, real-time quantitative polymerase chain reaction showed that the expression levels of let-7 family members in follicles and granulosa cells were similar to our microarray data, in which miRNAs let-7a, let-7b, let-7c, and let-7i were significantly decreased in early atretic and progressively atretic porcine ovary follicles compared with healthy follicles, while let-7g was highly expressed during follicle atresia. Furthermore, flow cytometric analysis and Hoechst33342 staining demonstrated that let-7g increased the apoptotic rate of cultured granulosa cells. In addition, let-7 target genes were predicted and annotated by TargetScan, PicTar, gene ontology and Kyoto encyclopedia of genes and genomes pathways. Our data provide new insight into the association between the let-7 miRNA family in granulosa cell programmed death.
\end{abstract}

\section{INTRODUCTION}

Ovarian follicles grow from primordial follicles into primary, secondary and tertiary follicles. Although follicle development is an ongoing process, atresia prevents follicles from ovulating to control reproductive activity. Previous studies have indicated that ovarian follicular atresia is mediated primarily by granulosa cell apoptosis (Tilly et al., 1991). Since then, additional studies have investigated the molecular mechanisms responsible for granulosa cell apoptosis during follicle atresia. Death-ligandreceptor-induced and mitochondria-controlled apoptotic pathways are the main hypotheses regarding the activation of granulosa cell apoptosis (Manabe et al., 2004). Tumor necrosis

Department of Animal Genetics, Breeding and Reproduction, College of Animal Science and Technology, Nanjing Agricultural University, Nanjing, 210095, China

*Correspondence: 2011105017@njau.edu.cn, liuhonglin@263.net

Received 12 May, 2014; revised 16 January, 2015; accepted 19 January, 2015; published online 31 March, 2015

Keywords: apoptosis, follicle atresia, let-7, MicroRNA, MiRNA, pig, stem-loop factor (TNF) alpha and receptors, fatty acid synthase (FAS) ligand (FasL) and receptors, and TNF-related apoptosis-inducing ligand (TRAIL), are death ligand receptors known to trigger apoptosis (Lin et al., 2010; Sasson et al., 2002; Wada et al., 2002). In mitochondrial apoptosis, members of the BCL-2 family, including $\mathrm{BAX}$ and $\mathrm{BAD}$, target the mitochondrial membrane to release cytochrome $c$, which assembles with Apaf-1 and pro-caspase- 9 (Li et al., 1997). This complex activates the caspase family of proteases signal cascade, ultimately resulting in cell death. In contrast, other BCL-2 family members, such as BCL-2 and BCL$\mathrm{XL}$, are anti-apoptotic, promoting cell survival through interactions with pro-apoptotic members of the BCL-2 family (MatsudaMinehata et al., 2006). Ultimately, the intracellular balance between pro- and anti-apoptotic factors determines cell fate.

MicroRNAs (miRNAs), which are approximately 22 nucleotides (nt) in length, have emerged as pivotal post-transcriptional regulators that cause gene silencing. MiRNAs regulate several biological and cellular processes, including cell development, proliferation and apoptosis (Dai et al., 2011). Mature miRNAs are incorporated into the miRNA induced-silencing complex (miRISC) with argonaute family proteins (AGOs), which target the $3^{\prime}$-untranslated region (UTR) of the target gene, causing mRNA decay in plants or protein degradation in mammals (Eulalio et al., 2009). In general, miRNAs recognize complementary binding sites in the coding region, as is the case for plants, or, in mammals, the mature miRNA partially complements the $3^{\prime}-U T R$, resulting in translational repression (Huntzinger and Izaurralde, 2011). MiRNA lethal-7 (let-7) was one of the first two miRNAs discovered in Caenorhabditis elegans that controls developmental timing (Pasquinelli et al., 2000; Reinhart et al., 2000). Let-7 family members are considered tumor suppressors (Trang et al., 2010). Furthermore, whether the let-7 family is involved in granulosa cell apoptosis is unknown. In 2010, Carletti et al. reported that miR-21 blocks apoptosis in murine granulosa cells, providing the first evidence that miRNAs play an important role in granulosa cell fate.

Our previous research identified differentially regulated miRNAs during follicular atresia in the porcine (Sus scrofa) ovary. Moreover, we demonstrated that one of these differentially expressed miRNAs (miR-26b) is involved in follicular atresia and granulosa cell apoptosis (Lin et al., 2012). However, the remainder of the miRNA chip data remained unexplored. Our current study is an extension of our previous microarray analysis. In this study, individual follicles were detached from porcine ovaries and divided into healthy $(\mathrm{H})$, early atretic $(E A)$, or progressively atretic (PA) follicles according to morphology and radioimmunoassay. Expression patterns of let-7 family mem- 
bers in $\mathrm{H}$, EA and PA ovary follicles were determined by quantitative real-time PCR. Moreover, let-7 mimics were transfected into cultured granulosa cells and their effects on apoptosis examined. This study provides valuable information for further functional investigation of granulosa cell apoptosis mediated by let-7 miRNAs.

\section{MATERIALS AND METHODS}

Isolation ovary follicle and granulosa cells

Mature porcine ovaries were collected at a local slaughterhouse (TianHuan, China). Porcine ovaries were transferred to the laboratory within $1 \mathrm{~h}$ (maintained above $30^{\circ} \mathrm{C}$ ) and washed by pre-warmed phosphate buffered saline (PBS, $\left.37^{\circ} \mathrm{C}\right)$ supplemented with penicillin $(100 \mathrm{lU} / \mathrm{ml})$, streptomycin $(100 \mu \mathrm{g} / \mathrm{ml})$. Follicles of 3-5 mm in diameter were separated and classified by their morphology as $\mathrm{H}$, EA or PA. Small scissors were used to cut the individual porcine ovary follicles and the cumulusoocyte complexes (COCs) were removed under a surgical dissecting microscope (SZ40, Olympus, Japan). Follicular fluid and granulosa cells, including mural granulosa cells, were collected into $1.5 \mathrm{ml}$ tubes and centrifuged at $3,000 \times g$ for $7 \mathrm{~min}$ to separate them from each other.

\section{Radioimmunoassay of 17b-estradiol (E2) and progesterone (P4) levels}

The follicular fluid was immediately sent to the General Hospital of the Nanjing Military Command to quantify the P4 and E2 levels using P4 and E2 radioimmunoassay kits (Beijing North Institute of Biological Technology).

\section{Re-analysis of $\mu$ ParafloTM miRNA microarray data}

In our previous study, 23 differentially expressed miRNAs were obtained under conditions of at least one signal value $>1000$ with an EA/H $>2$ or an EA/PA $<0.7$ and the role of miR-26b in granulose cell apoptosis was characterized (Lin et al., 2012). However, follicle atresia and granulosa cell apoptosis are highly orchestrated processes controlled by many extrinsic and intrinsic factors, and the miRNA molecular regulatory mechanisms in granulosa cell death during follicular atresia may involve multiple miRNAs. Therefore, we statistically analyzed the chip signals in the $\mathrm{H}, \mathrm{EA}$ and PA groups using a one-way ANOVA test and analyzed the expression patterns of let-7 family members systematically in this study. Moreover, TargetScan (http://www. targetscan.org/) and PicTar (http://pictar.mdc-berlin.de/) were used to predict let-7 target genes. Gene ontology (GO) was used to annotate the functions of let-7 target genes and Kyoto encyclopedia of genes and genomes (KEGG) pathway analysis indicated whether the target genes are involved in apoptotic processes.

Isolation of total RNA, design of Stem-loop RT primers and synthesis of miRNA first-strand cDNA

Follicles and granulosa cells whose P4/E2 values matched their morphology were selected to analyze the expression patterns of the let-7 family. The Trizol Reagent (Invitrogen, USA) was used to extract total RNA from $\mathrm{H}$, EA and PA follicles, respectively, following the manufacturer's instructions.

MiRNA let-7a/b/c/g/i stem-loop primers and forward primers were designed according to the method provided by Chen et al. (2005). Briefly, stem-loop primers for let-7 family mature sequences were designed independently. Considering that the continuous eight nucleotides that began from the $3^{\prime}$ region of the let-7 mature sequences was the most variable region within the highly conserved let-7 family, the stem-loop RT primers comprised a universal stem-loop sequence (5'-CTCAACTGGTGTCGTGGAGTCGGCAATTCAGTTGAG-3') and eight nucleotides that were the reverse complement to the last eight nucleotides of the let-7 family. The forward primers and universal reverse primers are presented in Table S1. The length of the designed products was $64 \mathrm{bp}$. PrimeScript ${ }^{\mathrm{TM}} \mathrm{RT}$ Master Mix (Takara, China) was used to synthesize the cDNA copy of the miRNA.

\section{PCR and TA cloning}

To evaluate the specificity of the stem-loop RT-PCR, we isolated total RNA from granulosa cells of individual porcine ovary follicle, regardless of their morphology. The total RNA from granulosa cells were used to test whether let-7 miRNA expressed in pig granulosa cells. MiRNA let-7a, let-7b, let-7c, let-7i and let-7g were reversed transcribed using a specific stem-loop primer. PCR was performed on a Thermal Cycler PCR Detection System. 4\% agarose gel and $20 \mathrm{bp}$ DNA ladder marker (Takara) was used to detect whether the lengths of the PCR products were as expected. It is difficult to purify such short DNA fragments from PCR mixtures; therefore, the let-7 family PCR products were directly inserted into vector pMD18-T, and transformed into $E$. coli $\mathrm{DH} 5 \alpha$ competent cells. The positive plasmids were sequenced by the Beijing Genomics Institution.

\section{Real-time PCR analysis}

Stem-loop RT-PCR based SYBR Green I Real-time PCR analysis was used to examine the expression patterns of let-7 family mature miRNAs in follicles or granulosa cells. Real-time quantitative PCR (qPCR) was performed on a Step-One Plus Real-Time PCR System (Applied Biosystems) with SYBR Premix ExTaq II (Takara) following the manufacturer's instructions. Analysis of each sample was repeated in triplicate. The miRNA expression fold change was calculated with the reference to the expression of the $\mathrm{U} 6$ gene using the ${ }^{2-\Delta \Delta} \mathrm{Ct}$ method.

\section{Cell culture and transfection}

To collect granulosa cells, we used syringes to puncture the follicles $(3-5 \mathrm{~mm})$. Granulosa cells were washed by preheated PBS $\left(37^{\circ} \mathrm{C}\right)$ twice and placed into $25 \mathrm{~cm}^{2}$ flask with culture medium DMEM/F12 (1:1) containing penicillin (100 IU/ml), streptomycin $(100 \mu \mathrm{g} / \mathrm{ml})$ and $15 \%$ fetal bovine serum in an incubator at $37^{\circ} \mathrm{C}$ and $5 \% \mathrm{CO}_{2}$. Let-7 mimics and negative controls (NC) were purchased from GenePharma (China). Let-

Table 1. Detection of $17 \mathrm{~b}$-estradiol (E2) and progesterone (P4) levels in follicles

\begin{tabular}{cccccc}
\hline & $\begin{array}{c}\text { Follicle } \\
\text { number }\end{array}$ & $\begin{array}{c}\text { Progesterone (P) } \\
(\mathrm{pg} / \mathrm{ml})\end{array}$ & $\begin{array}{c}\text { 17b-estradiol (E) } \\
(\mathrm{pg} / \mathrm{ml})\end{array}$ & $\mathrm{P} / \mathrm{E}$ Morphology \\
\hline $\mathrm{H}$ & 1 & 7450 & 1563.52 & 4.76 & $\mathrm{H}$ \\
& 2 & 5110 & 1575.37 & 3.24 & $\mathrm{H}$ \\
& 3 & 6250 & 1261.49 & 4.95 & $\mathrm{H}$ \\
\hline $\mathrm{EA}$ & 1 & 7493 & 1042.35 & 7.18 & $\mathrm{EA}$ \\
& 2 & 6610 & 1188.25 & 5.56 & $\mathrm{EA}$ \\
& 3 & 8420 & 889.37 & 9.46 & $\mathrm{EA}$ \\
\hline $\mathrm{PA}$ & 1 & 9753 & 510.48 & 19.11 & $\mathrm{PA}$ \\
& 2 & 12560 & 532.10 & 23.6 & $\mathrm{PA}$ \\
& 3 & 11760 & 471.76 & 24.9 & $\mathrm{PA}$ \\
\hline
\end{tabular}

$\mathrm{H}$ : The ratio of $\mathrm{P} / \mathrm{E}$ less than 5 was considered as $\mathrm{H}$;

$E A$ : The ratio of $P / E$ between $5-15$ was considered as $E A$;

$\mathrm{PA}$ : The ratio of $\mathrm{P} / \mathrm{E}$ more than 15 was considered as $\mathrm{PA}$ 
Health $(\mathbf{H})$
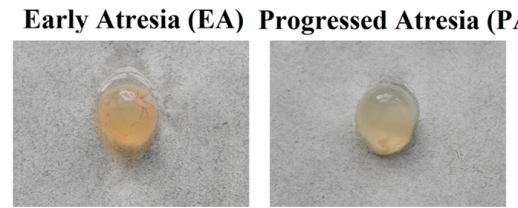

Fig. 1. Phenotypic characteristic of healthy, early atretic and progressively atretic porcine ovary follicles. $\mathrm{H}$, Healthy follicles. Healthy follicles had visible cumulus-oocyte complex (COC) and clear follicular fluid. EA, Early atretic follicles. Early atretic follicles had gradually separated $\mathrm{COC}$ and slightly turbid follicular fluid. PA, Progressively atretic follicles. Progressively atretic follicles had turbid follicular fluid and were barely vascularized.

7 mimics $(75 \mathrm{nM})$ and $\mathrm{NC}(75 \mathrm{nM})$ were transfected into granulosa cells using Lipofectamine 2000 (Invitrogen) following the manufacturer's protocol.

\section{Apoptosis assay}

Granulosa cells transfected with let-7 mimics or NC were harvested after $72 \mathrm{~h}$ incubation and washed in cold PBS. We use Annexin V-FITC Apoptosis Detection Kit (KeyGEN, China) to detect apoptosis of the transfected cells. After staining a cell population $\left(1-5 \times 10^{5}\right)$ with Annexin V-FITC and propidium iodide (PI), according to the manufacturer's instructions, we analyzed the stained granulosa cells by flow cytometry within $1 \mathrm{~h}$. Meanwhile, nuclei were first fixed in $4 \%$ paraformaldehyde for 1hour at room temperature, then stained with chromatin dye Hoechst33342 (Beyotime, China) for $1 \mathrm{~h}$ at $37^{\circ} \mathrm{C}$ after $72 \mathrm{~h}$ transfections with let-7 mimics. The changes of cellular morphology were confirmed under a confocal laser microscope (Carl Zeiss, Germany).

\section{Statistical analysis}

Three independent samples from porcine $\mathrm{H}$, EA, and PA ovary follicles were collected to analyze the expression patterns of let7 family. All statistical evaluation was performed using SPSS for windows version 18.0. All data were presented as the mean \pm SE. A one-way ANOVA test was used for multiple comparisons. A value of $P<0.05$ was deemed statistically significant, $P$ $<0.05$ was shown as ${ }^{*}, P<0.01$ was shown as ${ }^{* *}$, and $P<$ 0.001 was shown as ${ }^{\star \star *}$.

\section{RESULTS}

\section{Porcine ovary follicle classification}

On the basis of morphology, visible COCs (Bortul et al., 2003), clear follicular fluid, and well vascularized characterized $\mathrm{H}$ follicles. COCs and granulosa cells layer were somewhat separated and slightly turbid follicular fluid could be observed in EA follicles. PA follicles had turbid follicular fluid and were barely vascularized (Carson et al., 1979; Hay et al., 1976). The phenotype of $\mathrm{H}, \mathrm{EA}$ and PA follicles is shown in Fig. 1.

Meanwhile, the P4/E2 (P/E) value was used to confirm the classification of the follicles accurately (Manabe et al., 1996). A $\mathrm{P} / \mathrm{E}$ ratio less than 5 was considered $\mathrm{H}$. A P/E ratio between 5 and 15 was considered EA and > 15 was considered PA (Table.1) (Cheng et al., 2008). The individual follicles whose morphology matched with the P/E value were chosen for expression patterns analysis of let-7 family members.
$\boldsymbol{A}$
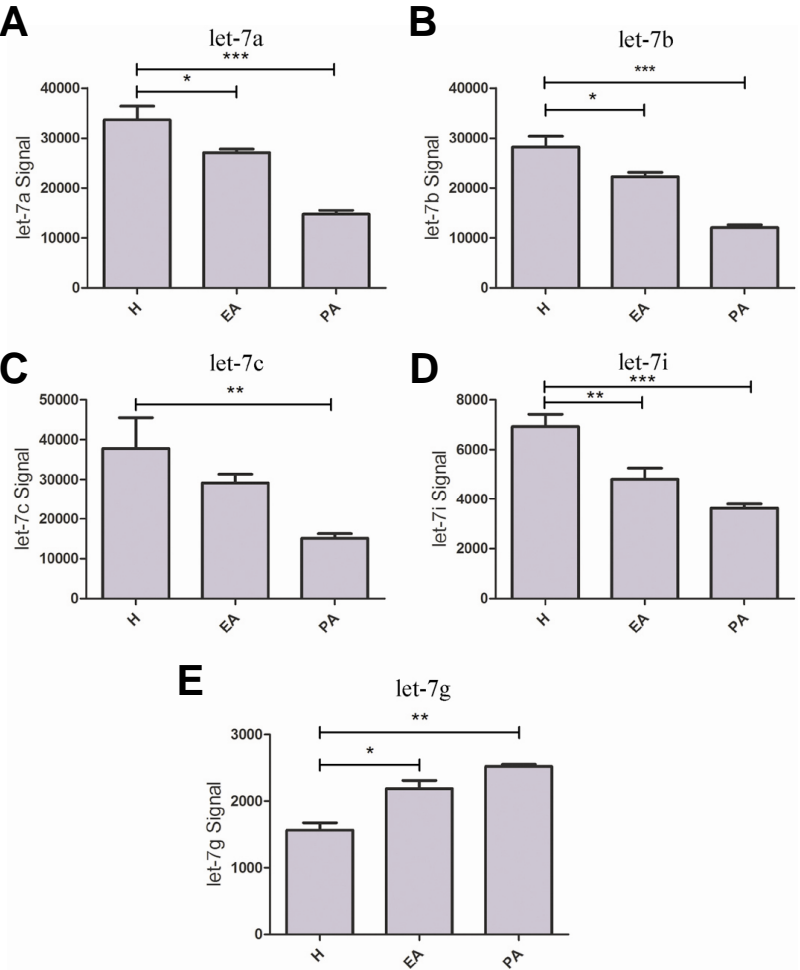

Fig. 2. Let-7 miRNA family expression signals during follicular atresia detected by an miRNA microarray assay. Mature let-7a $(A)$, let-7b $(B)$, let-7c $(C)$, and let-7g (D) microarray signals were determined in healthy $(H)$, early atretic $(E A)$, and progressively atretic $(P A)$ follicles. The expressions of let-7a, let-7b and let-7c were downregulated during follicle atresia, while, let-7g expression was upregulated (relative to $\mathrm{H}$ ). Data are presented as the mean $\pm \mathrm{SE}$. A one-way ANOVA test was used for multiple comparisons. $P<0.05$ is shown as ${ }^{*}, P<0.01$ is shown as ${ }^{* *}$, and $P<0.001$ is shown as ***

porcine mature let-7 miRNAs (let-7a, let-7c, let-7d, let-7e, let-7f, let-7g, and let-7i) have been published. Interestingly, our results showed that all these mature let-7 miRNAs signals could be detected in our chip data, adding let-7b. MiRNA let-7a, let-7b, let-7c and let-7i were significantly decreased in EA and PA porcine ovary follicles relative to $\mathrm{H}$ follicles. Interestingly, let-7g was significantly increased during the process of follicle atresia (Fig. 2).

\section{Specificity of let-7 family identified by Stem-loop RT-PCR}

To evaluate the specificity of Stem-loop RT-PCR for the let-7 family, we chose let-7a/b/c/g/i as candidates and PCR was conducted to amplify miRNA cDNA. Agarose gel electrophoresis confirmed the length of the PCR products (64 bp). As shown in Fig. 3A, all the PCR products were slightly above $60 \mathrm{bp}$, without hairpins or dimers. Furthermore, TA cloning and sequencing confirmed the let-7 family mature sequences. In eight successful sequencing samples, seven samples' sequences matched with let-7a mature sequences (Fig. 3B). Let-7b was successfully identified in six out of 11 sequenced samples (Fig. $3 C$ ), let-7c was successfully identified in eight out of nine sequenced samples (Fig. 3D), let-7i was successfully identified in six out of eight sequenced samples (Fig. 3E) and let-7g was successfully identified in 19 out of 21 sequenced samples (Fig. 3F). All the unmatched sequences did not match the other 
$\boldsymbol{A}$

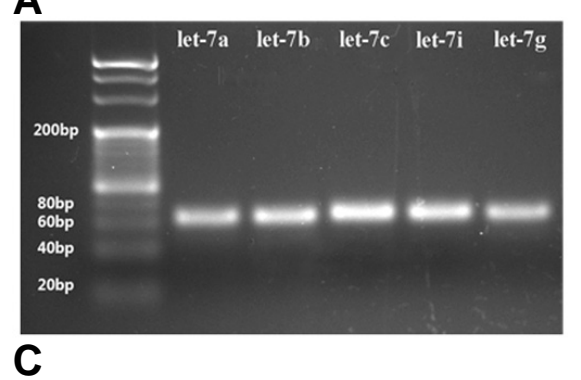

let-7b: TGAGGTAGTAGGTTGTGTGGTT

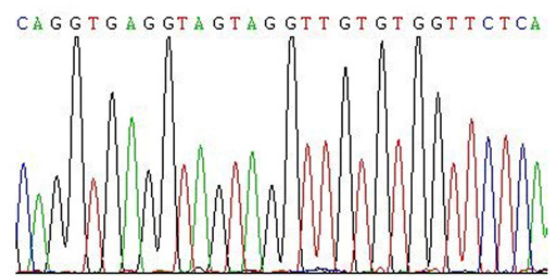

$E$

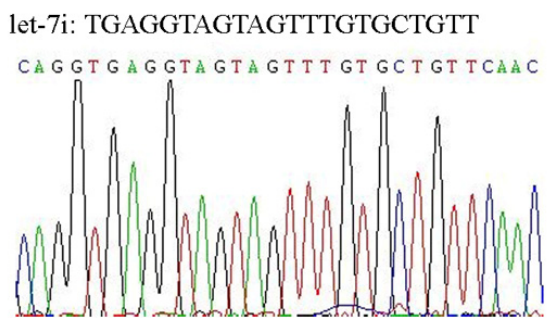

$B$

let-7a:TGAGGTAGTAGGTTGTATAGTT

CÀG GT GA G GT À GT À G GTT GTAT À GT TCTCÀ

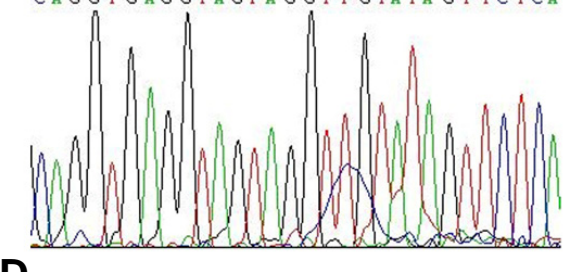

D
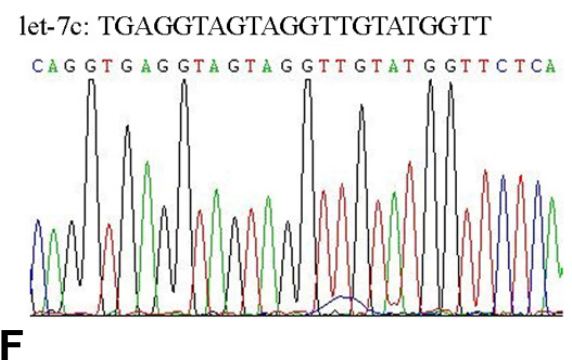

let-7g: TGAGGTAGTAGTTTGTACAGTT

CA G GT GA G GT A GTAGT T T GTACA GT T CTCA

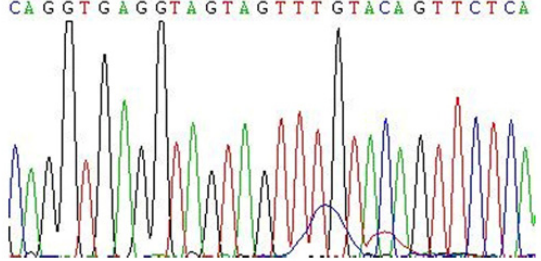

Fig. 3. Specific, quantitative detection of let-7 family by stem-loop RT-PCR and sequencing. Let-7 miRNA sequences were amplified by PCR and the PCR products were fractionated by electrophoresis on $4 \%$ agarose gels. Each band represented one member of the let-7 family. Let-7 family miRNAs were cloned into vector pMD18-T and their sequences determined (B-F). members of let-7 family.

\section{Expression profiling of let-7 family in $H, E A$, and PA ovary follicles}

We discriminated $\mathrm{H}$, EA and PA porcine ovary follicles by morphology and the ratio of Progesterone (P4)/17b-estradiol (E2) and extracted total RNA from each group. We then tested whether SYBR Green I real-time-PCR could quantify the let-7 family levels in these follicles. U6 snRNA was used as normalization control. The results are shown in Fig. 4. MiRNA let-7a, let-7b, let-7c and let-7i showed decreased abundance in EA and PA (Figs. 4A, 4B, 4C, 4D); however, let-7g was significantly increased in EA and PA relative to its expression in $\mathrm{H}$ (Fig. $4 \mathrm{E})$. These results suggested that the expression patterns of the let-7 family members mostly correlated with the miRNA microarray data. The exception was let-7c, whose mature form did not show significantly reduced expression in EA relative to $\mathrm{H}$ based on our microarray data (Fig. 2C); however, stem-loop real-time-PCR showed that the relative abundance of mature let-7c was much lower in EA than in $\mathrm{H}$ (Fig. 4C).

Expression analysis of let-7 Family in granulosa cells in response to porcine ovary follicular atresia

We extracted total RNA of granulosa cells from $\mathrm{H}$, EA and PA follicles to test whether let-7 family were expressed in granulosa cells. The results were consistent with the expression pattern of let-7 family in follicles and microarray (Fig. 5). Previous studies showed that apoptosis triggered granulosa cell death and apoptotic granulosa cells increased in number during follicular atresia (Hughes and Gorospe, 1991; Sugimoto et al., 1998). Our results suggested that let-7 family were associated with the process of follicular apoptosis.

\section{Apoptosis analysis}

To explore the function of let-7 family in cultured granulosa cells, we divided the let-7 family into two groups: let-7a/b/c/i and let7g. Let-7a, let-7b, let-7c and let-7i mimic were co-transfected into granulosa cells in vitro, while we transfected let-7g alone into cultured granulosa cells; NC (a scrambled sequence) was used as the normalized control. After $72 \mathrm{~h}$ incubation, we stained the transfected granulosa cells with Annexin V-FITC and propidium iodide (PI). The fluorescence-activated cell sorting (FACS) analyses are shown in Figs. 6A, 6B and 6C. The extent of apoptosis in granulosa cells transfected with the let-7g mimic increased significantly (Fig. 6D) relative to NC. This suggested that overexpression of let-7g could induce apoptosis in granulosa cells in vitro. In addition, typical apoptotic nuclei including nuclear shrinkage, chromatin condensation and fragmentation were detected by staining with Hoechst33342 when transfected with let-7g (Fig. 6E), which indicated those cells could be in the apoptosis processes.

\section{Target genes and functions of let-7 miRNA family}

Targets prediction, GO analysis and KEGG pathway analysis indicated that let-7a, let-7b, let-7c and let-7i co-regulated 496 genes (Fig. 7A), while let-7g was predicted to target 501 genes. Furthermore, the most relevant biological process involving the target genes of both let-7a/b/c/i (Fig. 7B) and let-7g (Fig. 7D) 

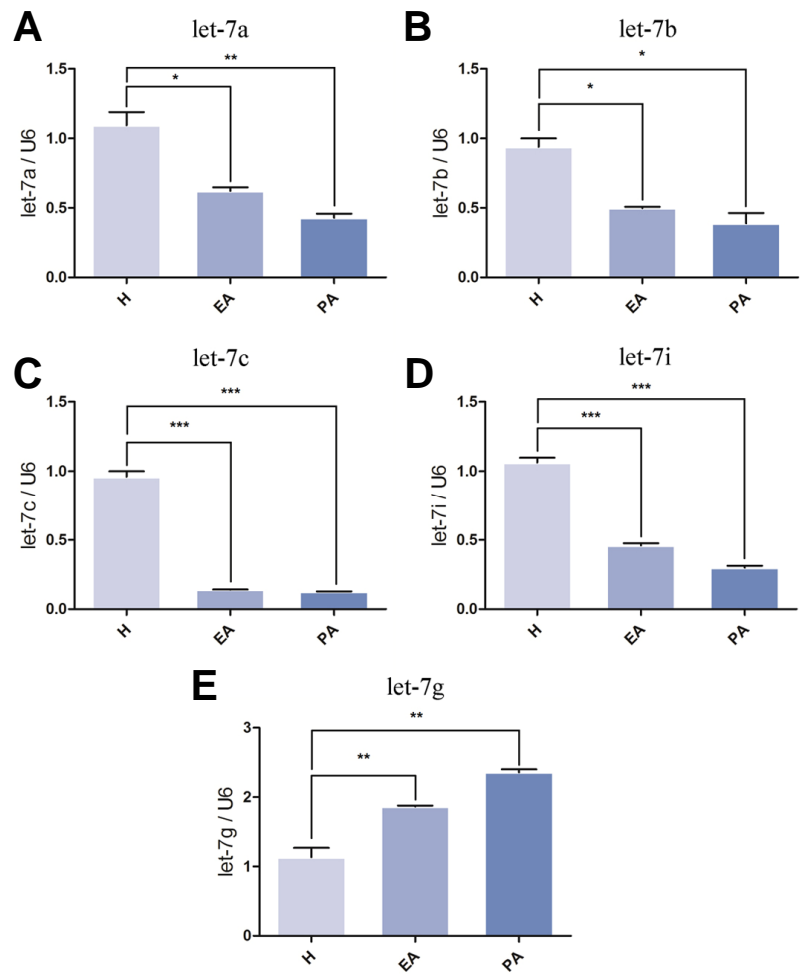

Fig. 4. Let-7 family expression patterns in $\mathrm{H}$, EA, and PA follicles. Detailed views of let-7a, let-7b, let-7c, let-7i and let-7g expression profiling in follicles undergoing atresia are presented in $(A)-(E)$, respectively. Total RNA was extracted from the whole $\mathrm{H}$, EA, and PA follicles for real-time PCR analysis. U6 snRNA was used as the normalization control. Data are presented as the mean $\pm \mathrm{SE}$. A one-way ANOVA test was used for multiple comparisons. $P<0.05$ is shown as ${ }^{*}, P<0.01$ is shown as ${ }^{* *}$, and $P<0.001$ is shown as ${ }^{* \star *}$.

was regulation of transcription, followed by positive regulation of signal transduction and posttranscriptional regulation of gene expression. These predicted targets of downregulated let7a/b/c/i (Fig. 7C) and let-7g (Fig. 7E) during follicular atresia may be involved in MAPK, TGF- beta and p53 signaling pathways, which affect cell proliferation, cell cycle or cell survival during follicle atresia.

\section{DISCUSSION}

According to morphological changes and differences in P4 and E2 levels, porcine ovary follicles were divided into healthy $(H)$, early atretic (EA), and progressively atretic (PA) follicles. Hormones (Hsueh et al., 1994), nutrient deprivation (Diskin et al., 2003) and oxidative stress (Tilly and Tilly, 1995) contribute to follicular fate. Moreover, in pigs, these factors affect breeding and reproduction, which are important economic traits for domestic animals. FSH, LH/hCG and estrogens suppress ovary follicle apoptosis (Chun et al., 1996). In contrast, gonadotropins, androgens and gonadal hormones are involved in follicular atresia (Craig et al., 2007; Hillier and Tetsuka, 1997). Moreover, the estrogen receptor (ER) inhibits miRNA processing, which is dependent on the RNA helicases p68/p72. In this process, the E2/ERa complex interacts with p68/p72 and dissociates Drosha from pri-miRNAs. ER-mediated inhibition of pri-miR-145
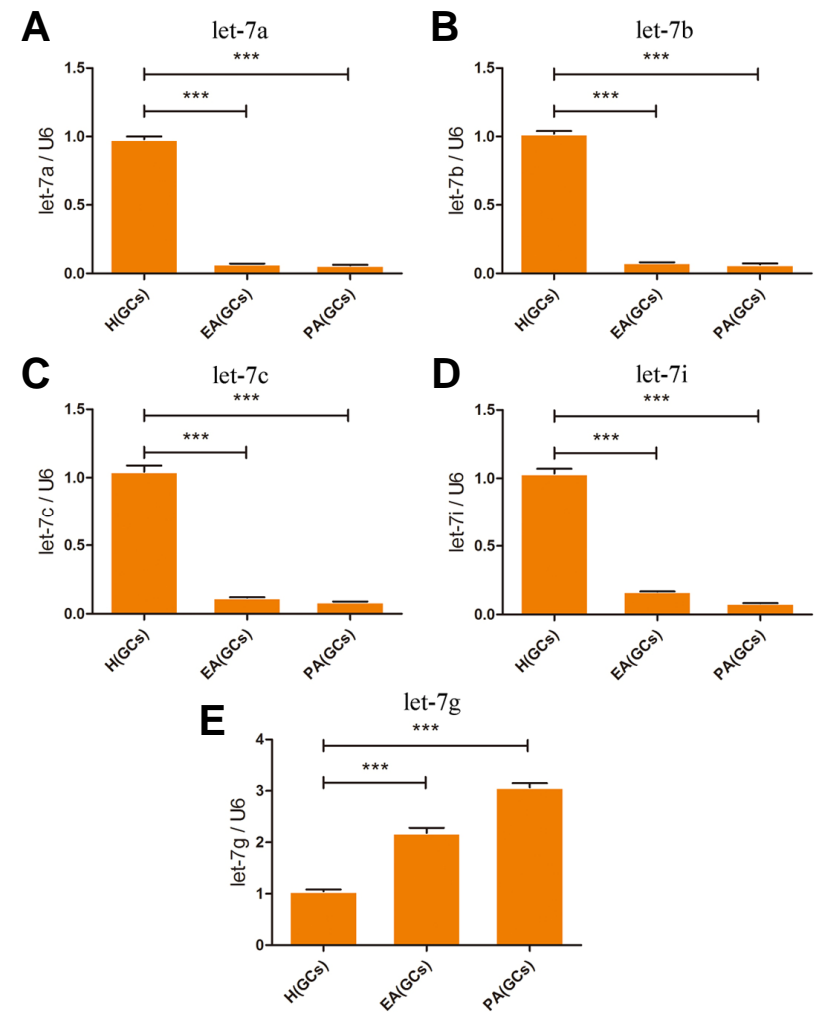

Fig. 5. Expression profiling of let-7 family in granulosa cells derived from in $\mathrm{H}, \mathrm{EA}$, and PA follicles. The relative abundance of let-7a, let$7 \mathrm{~b}$, let-7c, let-7i, let-7g in granulosa cells from $\mathrm{H}, \mathrm{EA}$ and PA follicles are shown in $(A)$ to $(E)$, respectively. The granulosa cells, but not the whole follicles, were used for expression patterns analysis of let 7 family members using real-time PCR. U6 snRNA was used as normalization control. Data are presented as mean \pm SE. A one-way ANOVA test was used for multiple comparisons. $P<0.05$ is shown as ${ }^{*}, P<0.01$ is shown as ${ }^{\star \star}$, and $P<0.001$ is shown as ${ }^{* \star}$.

and pri-miR-195 is stimulated by E2. Our previous microarray data showed that levels of these mature miRNAs were increased in follicle atresia, while E2 was reduced (Guthrie et al., 1995; Yamagata et al., 2009). Meanwhile, E2 suppresses the expression of mature let-7g though E2-ERa in MCF-7 cells (Qian et al., 2011). The relative abundance of mature let-7b in mouse endometrial cells increases after treatment with steroid hormones, including P4 or E2 (Fu et al., 2011). These studies suggested that hormonal signals also regulate the differential abundance of miRNAs during follicle atresia, and can activate or suppress intracellular apoptotic pathways. Therefore, use of a miRNA microarray was proposed to examine miRNA expression profiles among healthy $(H)$, early atretic $(E A)$, or progressively atretic (PA) porcine ovary follicles.

Interestingly, deep sequencing of adult Tibetan ovary and testis miRNA libraries indicated that let-7i, let-7f, let-7a, and let7c were represented in the top 10 unique miRNAs with the highest expression levels (Li et al., 2011), suggesting that they play important roles in reproductive system development.

Based on miRBase 21.0, the mature sequence of porcine let$7 i$ (ssc-let-7i) is $5^{\prime}$-UGAGGUAGUAGUUUGUGCU-3', and the mature sequence of human and mouse let-7i is $5^{\prime}-U G A G-$ GUAGUAGUUUGUGCUGUU-3'. According to miRBase, ssc- 
$\boldsymbol{A}$

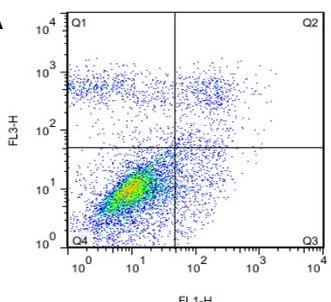

NI ${ }^{\mathrm{A}} \mathrm{H}$
$\boldsymbol{B}$

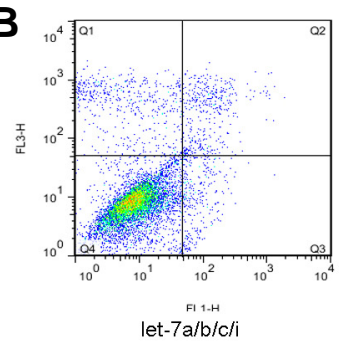

C

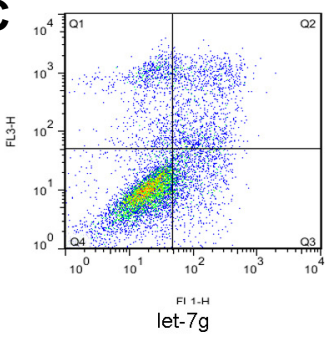

D

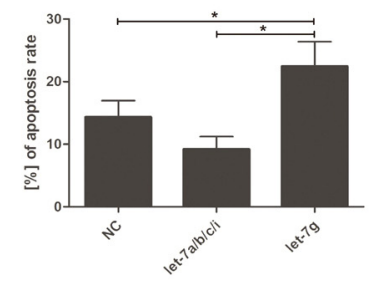

\section{$E$}

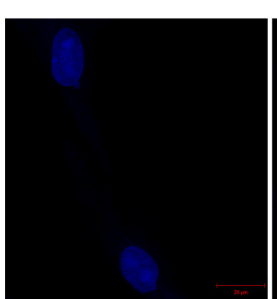

NC

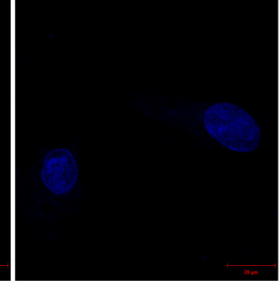

let-7 a/b/c/i

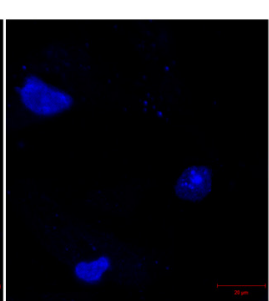

let-7g

Fig. 6. Analysis of apoptosis in cultured granulosa cells transfected with let-7 mimics. Granulosa cells transfected with negative control (NC) $(\mathrm{A})$, let-7a/b/c/i mimics $(\mathrm{B})$ or let-7g mimic $(\mathrm{C})$ were stained with Annexin $\mathrm{V}$ and PI. The apoptosis level of each group was analyzed by flow cytometry. Analysis of different apoptosis rates in NC, let-7a/b/c/i co-transfection group and let-7g transfection group (D). Data are presented as mean $\pm \mathrm{SE}$. A one-way ANOVA test was used for multiple comparisons. $P<0.05$ is shown as *. Morphological changes in granulosa cells transfected with NC, let-7a/b/c/i and let-7g mimics (from left to right) were stained with Hoechst33342 and images were obtained under a fluorescence microscope $(\mathrm{E})(\mathrm{bar}=20 \mu \mathrm{m})$.

B

negative regulation of macromolecule biosynthetic process positive regulation of biosynthetic process positive regulation of cellular biosynthetic process regulation of cell motion phosphate metabolic process
phosphorus metabolic process posttranscriptional regulation of gene expression positive regulation of signal transduction

\section{$D$}

negative regulation of macromolecule biosynthetic process positive regulation of biosynthetic process positive regulation of cellular biosynthetic process regulation of cell motion phosphate metabolic process phosphorus metabolic process
posttranscriptional regulation of gene expression
positive regulation of signal transduction positive regulation of signal transduction
regulation of transcription

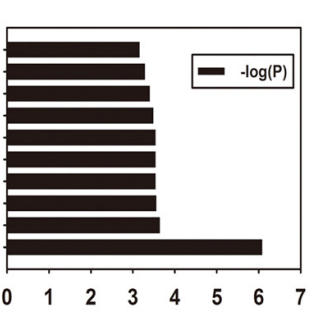

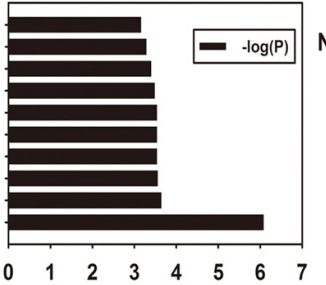

$E$

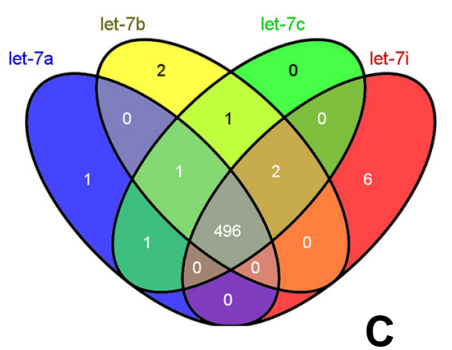

C

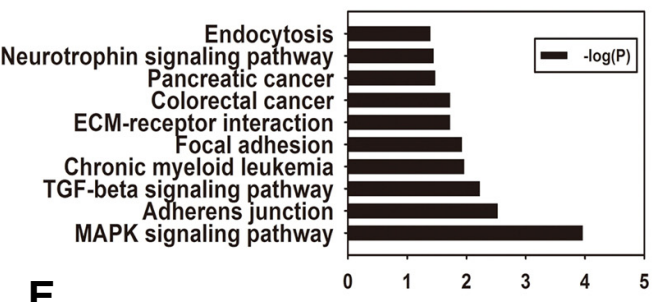

Endocytosis eurotrophin signaling pathway Pancreatic cance Colorectal cancer ECM-receptor interaction Chronic myeloid leukemia GF-beta signaling pathway Adherens junction MAPK signaling pathway

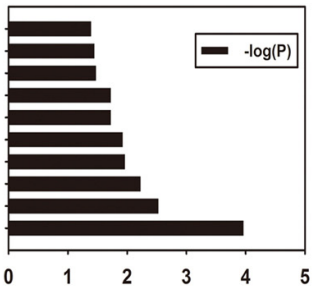

Fig. 7. Analysis of predicted let-7 target genes by number, function and signaling pathway. (A) Venn diagram analysis of the relationship between predicted let-7a/b/c/i target genes. (B) Main biological processes of predicted let-7a/b/c/i target genes based on $\mathrm{GO}$ analysis. (C) Main pathways involving predicted let-7a/b/c/i target genes based on KEGG pathway analysis. (D) Main biological processes of predicted let-7g target genes. (E) Main pathways involving predicted let-7g target genes. -Log10 values ( $P$ values), representing significant data from $G O$ and KEGG pathway analyses, are presented in the form of a histogram (SigmaPlot, Systat Software, USA). 
let-7i is recorded as a 19 nt sequence that lacks three nucleotides (GUU) at the $3^{\prime}$ end. Nevertheless, in 2010, Xie et al. identified microRNAs from porcine skeletal muscle and found that the length of ssc-let-7i was 21 nt (Xie et al., 2010). In the same year, $\mathrm{Li}$ et al. used whole body tissue from pig or a mixture of 60 tissue types to identify the microRNAome of porcine preand postnatal development. They showed that the most abundant microRNA for ssc-let-7i was 22 nt in length, consistent with nearly all mammalian mature let-7i in miRBase (Li et al., 2010). Further, Lian et al. profiled porcine testes using Solexa deep sequencing and found that the most abundant ssc-let-7i was 22 nt in length, similar to the results of Li et al. (Lian et al., 2012). Thus, the ssc-let-7i sequence remains disputed. Our mature let-7i sequencing results indicate that Ssc-let-7i is most likely 22 nt in length.

According to our prediction, let-7a/b/c/i may target TP53, CASP3 and FAS to prevent apoptosis, whereas let-7g may induce apoptosis by binding to CCND2 or BCL-XL. TP53, CASP3, FAS, CCND2, and BCL-XL represent pro- or anti-apoptotic genes that regulate granulosa cell fate. It has been reported that let-7a suppresses therapeutics-induced cancer cell death by targeting CASP3 (Tsang and Kwok, 2008). Additionally, let$7 \mathrm{a}$ and let-7b contribute to survival signaling by repressing interleukin (IL)-6 in human hepatocellular cancer stem cells (Meng et al., 2012). However, let-7g inhibits BCL-XL expression to promote sorafenib-induced apoptosis in human hepatocellular carcinoma (Shimizu et al., 2010). Notably, let-7g (Fig. 7E) may also function as a potent antagonist regulating the same pathways as let-7a/b/c/i (Fig. $7 \mathrm{C}$ ). However, further research is required to identify let-7 family functions, target genes, and associated signal pathways. Moreover, it is possible that the let-7 miRNA family forms a conserved regulatory network during granulosa cell apoptosis, similar to the conserved p53 regulatory network mediated by miRNA-125b (Le et al., 2011). However, the mechanisms of let-7 miRNA family mediated regulation of porcine ovarian follicular atresia require further validation.

Note: Supplementary information is available on the Molecules and Cells website (www.molcells.org).

\section{ACKNOWLEDGMENTS}

This work was supported by the National Natural Science Foundation of China (31172187).

\section{REFERENCES}

Bortul, R., Tazzari, P.L., Cappellini, A., Tabellini, G., Billi, A.M. Bareggi, R., Manzoli, L., Cocco, L., and Martelli, A.M. (2003). Constitutively active Akt1 protects HL60 leukemia cells from TRAIL-induced apoptosis through a mechanism involving NFkappaB activation and $\mathrm{cFLIP(L)}$ up-regulation. Leukemia 17, 379-389.

Carletti, M.Z., Fiedler, S.D., and Christenson, L.K. (2010). MicroRNA 21 blocks apoptosis in mouse periovulatory granulosa cells. Biol. Reprod. 83, 286-295.

Carson, R.S., Findlay, J.K., Burger, H.G., and Trounson, A.O. (1979). Gonadotropin receptors of the ovine ovarian follicle during follicular growth and atresia. Biol. Reprod. 21, 75-87.

Chen, C., Ridzon, D.A., Broomer, A.J., Zhou, Z., Lee, D.H., Nguyen, J.T., Barbisin, M., Xu, N.L., Mahuvakar, V.R., and Andersen, M.R. (2005). Real-time quantification of microRNAs by stemloop RT-PCR. Nucleic Acids Res. 33, e179.

Cheng, Y., Maeda, A., Goto, Y., Matsuda, F., Miyano, T., Inoue, N., Sakamaki, K., and Manabe, N. (2008). Changes in expression and localization of X-linked inhibitor of apoptosis protein (XIAP) in follicular granulosa cells during atresia in porcine ovaries. $\mathrm{J}$. Reprod. Dev. 54, 454-459.
Chun, S.Y., Eisenhauer, K.M., Minami, S., Billig, H., Perlas, E., and Hsueh, A.J. (1996). Hormonal regulation of apoptosis in early antral follicles: follicle-stimulating hormone as a major survival factor. Endocrinology 137, 1447-1456.

Craig, J., Orisaka, M., Wang, H., Orisaka, S., Thompson, W., Zhu, C., Kotsuji, F., and Tsang, B.K. (2007). Gonadotropin and intraovarian signals regulating follicle development and atresia: the delicate balance between life and death. Front. Biosci. 12, 36283639.

Dai, L., Tsai-Morris, C.H., Sato, H., Villar, J., Kang, J.H., Zhang, J., and Dufau, M.L. (2011). Testis-specific miRNA-469 up-regulated in gonadotropin-regulated testicular RNA helicase (GRTH/ DDX25)-null mice silences transition protein 2 and protamine 2 messages at sites within coding region: implications of its role in germ cell development. J. Biol. Chem. 286, 44306-44318.

Diskin, M.G., Mackey, D.R., Roche, J.F., and Sreenan, J.M. (2003). Effects of nutrition and metabolic status on circulating hormones and ovarian follicle development in cattle. Anim. Reprod. Sci. 78, 345-370.

Eulalio, A., Huntzinger, E., Nishihara, T., Rehwinkel, J., Fauser, M., and Izaurralde, E. (2009). Deadenylation is a widespread effect of miRNA regulation. RNA 15, 21-32.

Fu, T.Y., Lin, C.T., and Tang, P.C. (2011). Steroid hormoneregulated let-7b mediates cell proliferation and basigin expression in the mouse endometrium. J. Reprod. Dev. 57, 627635.

Guthrie, H.D., Cooper, B.S., Welch, G.R., Zakaria, A.D., and Johnson, L.A. (1995). Atresia in follicles grown after ovulation in the pig: measurement of increased apoptosis in granulosa cells and reduced follicular fluid estradiol-17 beta. Biol. Reprod. 52, 920-927.

Hay, M.R., Cran, D.G., and Moor, R.M. (1976). Structural changes occurring during atresia in sheep ovarian follicles. Cell Tissue Res. 169, 515-529.

Hillier, S.G., and Tetsuka, M. (1997). Role of androgens in follicle maturation and atresia. Baillieres Clin. Obstet. Gynaecol. 11, 249-260.

Hsueh, A.J., Billig, H., and Tsafriri, A. (1994). Ovarian follicle atresia: a hormonally controlled apoptotic process. Endocr. Rev. 15, 707-724.

Hughes, F.M., Jr., and Gorospe, W.C. (1991). Biochemical identification of apoptosis (programmed cell death) in granulosa cells: evidence for a potential mechanism underlying follicular atresia. Endocrinology 129, 2415-2422.

Huntzinger, E., and Izaurralde, E. (2011). Gene silencing by microRNAs: contributions of translational repression and mRNA decay. Nat. Rev. Genet. 12, 99-110.

Le, M.T., Shyh-Chang, N., Khaw, S.L., Chin, L., Teh, C., Tay, J. O'Day, E., Korzh, V., Yang, H., Lal, A., et al. (2011). Conserved regulation of p53 network dosage by microRNA-125b occurs through evolving miRNA-target gene pairs. PLoS Genet. 7, e1002242.

Li, P., Nijhawan, D., Budihardjo, I., Srinivasula, S.M., Ahmad, M., Alnemri, E.S., and Wang, X. (1997). Cytochrome c and dATPdependent formation of Apaf- $1 /$ caspase- 9 complex initiates an apoptotic protease cascade. Cell 91, 479-489.

Li, M., Xia, Y., Gu, Y., Zhang, K., Lang, Q., Chen, L., Guan, J., Luo, Z., Chen, H., Li, Y., et al., (2010). MicroRNAome of porcine preand postnatal development. PLoS One 5, e11541.

Li, M., Liu, Y., Wang, T., Guan, J., Luo, Z., Chen, H., Wang, X., Chen, L., Ma, J., Mu, Z., et al. (2011). Repertoire of porcine microRNAs in adult ovary and testis by deep sequencing. Int. J. Biol. Sci. 7, 1045-1055.

Lian, C., Sun, B., Niu, S., Yang, R., Liu, B., Lu, C., Meng, J., Qiu, Z., Zhang, L., and Zhao, Z. (2012). A comparative profile of the microRNA transcriptome in immature and mature porcine testes using Solexa deep sequencing. FEBS J. 279, 964-975.

Lin, P.F., Hao, Y.B., Guo, H.L., Liu, H.L., and Rui, R. (2010). Role of Fas/FasL on apoptosis of porcine follicular granulosa cells derived from isolated follicles during culture in vitro. Dongwuxue Yanjiu 31, 268-274.

Lin, F., Li, R., Pan, Z.X., Zhou, B., Yu de, B., Wang, X.G., Ma, X.S. Han, J., Shen, M., and Liu, H.L. (2012). miR-26b promotes granulosa cell apoptosis by targeting ATM during follicular atresia in porcine ovary. PLoS One 7, e38640.

Manabe, N., Imai, Y., Ohno, H., Takahagi, Y., Sugimoto, M., and 
Miyamoto, H. (1996). Apoptosis occurs in granulosa cells but not cumulus cells in the atretic antral follicles in pig ovaries. Experientia 52, 647-651.

Manabe, N., Goto, Y., Matsuda-Minehata, F., Inoue, N., Maeda, A. Sakamaki, K., and Miyano, T. (2004). Regulation mechanism of selective atresia in porcine follicles: regulation of granulosa cell apoptosis during atresia. J. Reprod. Dev. 50, 493-514.

Matsuda-Minehata, F., Inoue, N., Goto, Y., and Manabe, N. (2006) The regulation of ovarian granulosa cell death by pro- and antiapoptotic molecules. J. Reprod. Dev. 52, 695-705.

Meng, F., Glaser, S.S., Francis, H., DeMorrow, S., Han, Y., Passarini, J.D., Stokes, A., Cleary, J.P., Liu, X., Venter, J., et al. (2012). Functional analysis of microRNAs in human hepatocellular cancer stem cells. J. Cell. Mol. Med. 16, 160-173.

Pasquinelli, A.E., Reinhart, B.J., Slack, F., Martindale, M.Q., Kuroda, M.I., Maller, B., Hayward, D.C., Ball, E.E., Degnan, B., Muller, P., et al. (2000). Conservation of the sequence and temporal expression of let-7 heterochronic regulatory RNA. Nature 408 , 86-89.

Qian, P., Zuo, Z., Wu, Z., Meng, X., Li, G., Wu, Z., Zhang, W., Tan, S., Pandey, V., Yao, Y., et al. (2011). Pivotal role of reduced let$7 \mathrm{~g}$ expression in breast cancer invasion and metastasis. Cancer Res. 71, 6463-6474.

Reinhart, B.J., Slack, F.J., Basson, M., Pasquinelli, A.E., Bettinger, J.C., Rougvie, A.E., Horvitz, H.R., and Ruvkun, G. (2000). The 21-nucleotide let-7 RNA regulates developmental timing in Caenorhabditis elegans. Nature 403, 901-906.

Sasson, R., Winder, N., Kees, S., and Amsterdam, A. (2002). Induction of apoptosis in granulosa cells by TNF alpha and its attenuation by glucocorticoids involve modulation of Bcl-2. Biochem. Biophys. Res. Commun. 294, 51-59.

Shimizu, S., Takehara, T., Hikita, H., Kodama, T., Miyagi, T., Hosui, A Tatsumi, T., Ishida, H., Noda, T., Nagano, H., et al. (2010). The let-7 family of microRNAs inhibits Bcl-xL expression and potentiates sorafenib-induced apoptosis in human hepatocellular carcinoma. J. Hepatol. 52, 698-704.

Sugimoto, M., Manabe, N., Kimura, Y., MYOUMOTO, A., IMAI, Y., OHNO, H. and MIYAMOTO, $\mathrm{H}$. (1998). Ultrastructural changes in granulosa cells in porcine antral follicles undergoing atresia indicate apoptotic cell death. J. Reprod. Dev. 44, 7-14.

Tilly, J.L., Kowalski, K.I., Johnson, A.L., and Hsueh, A. (1991). Involvement of apoptosis in ovarian follicular atresia and postovulatory regression. Endocrinology 129, 2799-2801.

Tilly, J.L., and Tilly, K.I. (1995). Inhibitors of oxidative stress mimic the ability of follicle-stimulating hormone to suppress apoptosis in cultured rat ovarian follicles. Endocrinology 136, 242-252.

Trang, P., Medina, P.P., Wiggins, J.F., Ruffino, L., Kelnar, K., Omotola, M., Homer, R., Brown, D., Bader, A.G., Weidhaas, J.B., et al. (2010). Regression of murine lung tumors by the let-7 microRNA. Oncogene 29, 1580-1587.

Tsang, W.P., and Kwok, T.T. (2008). Let-7a microRNA suppresses therapeutics-induced cancer cell death by targeting caspase-3. Apoptosis 13, 1215-1222.

Wada, S., Manabe, N., Nakayama, M., Inou, N., Matsui, T., and Miyamoto, H. (2002). TRAlL-decoy receptor 1 plays inhibitory role in apoptosis of granulosa cells from pig ovarian follicles. $\mathrm{J}$. Vet. Med. Sci. 64, 435-439.

Xie, S.S., Huang, T.H., Shen, Y., Li, X.Y., Zhang, X.X., Zhu, M.J., Qin, H.Y. and Zhao, S.H. (2010). Identification and characterization of microRNAs from porcine skeletal muscle. Anim. Genet. 41, 179-190.

Yamagata, K., Fujiyama, S., Ito, S., Ueda, T., Murata, T., Naitou, M., Takeyama, K., Minami, Y., O'Malley, B.W., et al. (2009). Maturation of microRNA is hormonally regulated by a nuclear receptor. Mol. Cell. 36, 340-347. 